\title{
STUDIES OF THE GALACTIC BULGES USING THE POST-THEORETICAL MASS METHOD
}

\author{
DORU MARIAN SURAN and NEDELIA ANTONIA POPESCU \\ Astronomical Institute of Romanian Academy, Str. Cutitul de \\ Argint, No. 5, 75212 Bucharest 28, ROMANIA
}

\begin{abstract}
The results of a new method, Post Theoretical Mass Method (PTM) are presented in order to investigate radial pulsating stars (RPS) in galaxies. Further implications for stellar and galactic astrophysics are also discussed.
\end{abstract}

Key Words: radial pulsating stars, galaxies-components.

\section{Introduction}

In the present work we try to determine the properties of different galactic components using RPS as tracers. Detailed calculations are made for disk (Cepheids in our Galaxy) and halo (RR Lyraes in two $\mathrm{dE}$ satellites of M31), in order to determine the chemical structure (radial and transverse gradients) of galaxies. Because bulges are transient component between disk and halo, this method can be very atractive to investigate them.

\section{Observations}

In the CEPHEIDS program we investigate stars from the galactic disk ( $3 \mathrm{kpc} \leq \mathrm{d} \leq 15 \mathrm{kpc})$ with low period ( $1 \mathrm{~d} \leq \mathrm{P} \leq 10 \mathrm{~d})$ of intermediate mass ( $3 \mathrm{MO} \leq \mathrm{M} \leq 11 \mathrm{MO})$ in the 2 -nd and $3-\mathrm{rd}$ crossing of the instability band. We have 10 Cepheids for which was determined $(\mathrm{P},\langle\mathrm{B}\rangle-\langle\mathrm{V}\rangle)$. Also the data are supplemented by photometrical and spectroscopical results for more than 50 Cepheids.

In the RR LYRAES program we investigate 10 stars from the halo of two dE's (NGC 185,147) with periods $0.3 d \leq P \leq 0.8 d$ (RRab)with amplitudes $A V \geq 1$, no M31 contaminations, for which we obtain $(\mathrm{P}, \mathrm{Ag})$.

\section{Theory}

Our method is based on the concept of PTM (Suran 1985) and was largely dicussed in Suran (1991) (see his eq. [15]) where the third relation is used in the form of six-parameters relation $(\mathrm{P}-\mathrm{L}-\mathrm{M}-\mathrm{Te}-\mathrm{Y}-\mathrm{Z})$. 
Solutions of PTM method implie:

$[\mathrm{P},\langle\mathrm{B}\rangle-\langle\mathrm{V}\rangle] \quad(\mathrm{Cep})$

\}$===============\Rightarrow\left[\left(\mathrm{M}, \mathrm{L}, \mathrm{T}_{\mathrm{e}}\right),(\langle\mathrm{Y}\rangle, \mathrm{Z})\right]$

$\left[P, \quad A_{g}\right](R R \operatorname{Lyr})\left(1, \kappa\right.$, eos, $\left.H_{p u l s}\right)\left(a_{0}, ., f\right)$

\section{Calibrations}

In the CEPHEIDS program we use the (P-L-M) relation in the form of Becker, Iben Tuggle (1977). The temperature relation is in the form:

$$
\log \mathrm{T}_{\mathrm{e}}=3.886-0.175\left(\langle\mathrm{~B}\rangle_{0}-\langle\mathrm{V}\rangle_{0}\right), \mathrm{E}_{\mathrm{B}-\mathrm{V}}=\mathrm{E}_{\mathrm{J}}
$$

with an error limit $\Delta \mathrm{Te} \leq \pm 100^{\circ} \mathrm{K}$. We use $\langle\mathrm{Y}\rangle=0.28$.

In the RR LYRAES program we use the full 6-parameters relation (Suran 1992):

$$
\log L=0.81 \log M-5.97+0.595 \log \mathrm{P}+2.07 \log \mathrm{T}_{\mathrm{e}}^{-0.05[\mathrm{Fe} / \mathrm{H}]}
$$

For the determination of temperatures we use the relation:

$$
\log \mathrm{T}_{\mathrm{e}}=0.05 \mathrm{~A}_{\mathrm{g}}+3.774
$$

with a limiting error of $\Delta \mathrm{Te} \leq \pm 200^{\circ} \mathrm{K}$. We use $\langle\mathrm{Y}\rangle=0.23$.

\section{Results}

For the CEPHEIDS program we obtain two parameters linear relations (Suran 1985) and a true chemical gradient for Cepheids in the disk of the Galaxy of: $-0.06 / \mathrm{kpc}$.

In the RR LYRAES program we obtain estimations of the chemical structure of the two dE's $([\mathrm{Fe} / \mathrm{H}]=-1.77$ for NGC185 and respectively -1.31 for NGC 147).

\section{References}

Becker, S. A. , Iben, I. , Tuggle, R. S. 1977, Ap. J. , 218, 533;

Suran, M. D. 1985, Topics in Astronomy and Astrophys., 1, 1;

Suran, M.D. 1992, in Proceedings ofESO/EIPC Workshop on Early Type Galaxies, Marciana Marina (in press). 